\title{
Casimir Effect for Gauge Fields in Spaces with Negative Constant Curvature
}

\author{
A. A. Bytsenko \\ Departamento de Física, Universidade Estadual de Londrina, \\ Caixa Postal 6001, Londrina-Paraná, Brazil \\ E-mail address: abyts@uel.br \\ M. E. X. Guimarães \\ Departamento de Matemática, \\ Universidade de Brasilia, Brasilia, DF, Brazil \\ E-mail address: marg@unb.br \\ V. S. Mendes \\ Departamento de Física, Universidade Estadual de Londrina, \\ Caixa Postal 6001, Londrina-Paraná, Brazil \\ E-mail address: vsmendes@yahoo.com.br
}

(Dated: September, 2004)

\begin{abstract}
We consider gauge theories based on abelian $p$-forms on real compact hyperbolic spaces. Using the zeta-function regularization method and the trace tensor kernel formula, we determine explicitly an expression for the vacuum energy (Casimir energy) corresponding to skew-symmetric tensor fields. It is shown that the topological component of the Casimir energy for co-exact forms on even-dimensional spaces, associated with the trivial character, is always negative. We infer on the possible cosmological consequences of this result.

PACS numbers: $04.70 . \mathrm{Dy}, 11.25 . \mathrm{Mj}$
\end{abstract}

\section{INTRODUCTION}

The topological Casimir effect for scalar (or spinor) fields on spaces of form $\Gamma \backslash \widetilde{X}$, where $\Gamma$ is a discrete group acting on manifold $\widetilde{X}$, has become a very exciting and important issue in areas of quantum field theory and cosmology [1-9]. Initial evaluation of the Casimir energy has been given for $\tilde{X}=\mathbb{R}^{N}, \mathbb{S}^{N}$. In [7-15], the calculation involves the case in which $\tilde{X}$ is a Lobachevsky real hyperbolic space.

Maximally symmetric spaces, such as the hyperbolic spaces, play very important in supergravity [16], in superstring theory 17| and definitely plays a crucial role in cosmology [18, 19, 20]. Besides, hyperbolic space forms are examples of a general noncompact irreducible rank one symmetric space the proper and outlook mathematical machinery could be available. In this paper we present a decomposition of the Hodge Laplacian and the tensor kernel trace formula for free generalized gauge fields ( $p$-forms) on real hyperbolic space forms. The main ingredient required is a type of differential form structure on the physical, auxiliary, or ghost variables. We evaluate spectral functions and the Casimir effect associated with physical degrees of freedom of the Hodgede Rham operators on $p$-forms. Let $\omega_{p}, \varphi_{p}$ be exterior differential $p$-forms; then, the invariant inner product is defined by $\left(\omega_{p}, \varphi_{p}\right) \stackrel{\text { def }}{=} \int_{\tilde{X}} \omega_{p} \wedge * \varphi_{p}$. Under the action of the Hodge * operator the following properties for forms hold: $\omega_{p}=(-1)^{p(n-p)} \omega_{p}$, and $d d=\delta \delta=$ $0, \delta=(-1)^{n p+n+1} * d *$. The operators $d$ and $\delta$ are adjoint to each other with respect to this inner product for $p$-forms: $\left(\delta \omega_{p}, \varphi_{p}\right)=\left(\omega_{p}, d \varphi_{p}\right)$. In quantum field theory the Lagrangian associated with $\omega_{p}$ takes the form: $d \omega_{p} \wedge$ $* d \omega_{p}$ (gauge field), $\delta \omega_{p} \wedge * \delta \omega_{p} \quad($ co - gauge field $)$. The Euler-Lagrange equations, supplied with the gauge, give: $\mathfrak{L}_{p} \omega_{p}=0, \delta \omega_{p}=0$ (Lorentz gauge); $\mathfrak{L}_{p} \omega_{p}=$ $0, d \omega_{p}=0$ (co-Lorentz gauge). These Lagrangians give possible representation of tensor fields or generalized abelian gauge fields.

As an application, we evaluate the Casimir effect associated with topologically inequivalent configurations of Abelian co-exact forms on real compact hyperbolic manifolds.

\section{THE TRACE FORMULA APPLIED TO THE TENSOR KERNEL}

Let us consider an $N$-dimensional compact real hyperbolic space $X_{\Gamma}$ with universal covering $\widetilde{X}$ and fundamental group $\Gamma$. We can represent $\widetilde{X}$ as the symmetric space $G / K$, where $G=S O_{1}(N, 1)$ and $K=S O(N)$ is a maximal compact subgroup of $G$. Then we regard $\Gamma$ as a discrete subgroup of $G$ acting isometrically on $\widetilde{X}$, and we take $X_{\Gamma}$ to be the quotient space by that action: $X_{\Gamma}=\Gamma \backslash \tilde{X}=\Gamma \backslash G / K$. Let $\operatorname{Vol}(\Gamma \backslash G)$ denote the integral of the constant function $\mathbb{I}$ on $\Gamma \backslash G$ with respect to the $G$-invariant measure on $\Gamma \backslash G$ induced by $d x$, $\operatorname{Vol}(\Gamma \backslash G)=\int_{\Gamma \backslash G} \mathbb{I} d x$. For $0 \leq p \leq N-1$ the Fried trace 
formula applied to the heat kernel $\mathcal{K}_{t}=e^{-t \mathfrak{L}_{p}}$ holds [21]:

$$
\begin{aligned}
\operatorname{Tr}\left(e^{-t \mathfrak{L}_{p}}\right) & =I_{\Gamma}^{(p)}\left(\mathcal{K}_{t}\right)+I_{\Gamma}^{(p-1)}\left(\mathcal{K}_{t}\right) \\
& +H_{\Gamma}^{(p)}\left(\mathcal{K}_{t}\right)+H_{\Gamma}^{(p-1)}\left(\mathcal{K}_{t}\right),
\end{aligned}
$$

where $I_{\Gamma}^{(p)}\left(\mathcal{K}_{t}\right), H_{\Gamma}^{(p)}\left(\mathcal{K}_{t}\right)$ are the identity and hyperbolic orbital integrals respectively:

$$
\begin{aligned}
I_{\Gamma}^{(p)}\left(\mathcal{K}_{t}\right) & \stackrel{\text { def }}{=} \frac{\chi(1) \operatorname{Vol}(\Gamma \backslash G)}{4 \pi} \\
& \times \int_{\mathbb{R}} \mu_{\sigma_{p}}(r) e^{-t\left(r^{2}+p+\rho_{0}^{2}\right)} d r \\
H_{\Gamma}^{(p)}\left(\mathcal{K}_{t}\right) \stackrel{\text { def }}{=} & \frac{1}{\sqrt{4 \pi t}} \sum_{\gamma \in C_{\Gamma}-\{1\}} \frac{\chi(\gamma)}{j(\gamma)} t_{\gamma} C(\gamma) \chi_{\sigma_{p}}\left(m_{\gamma}\right) \\
& \times e^{-t\left(\rho_{0}^{2}+p\right)-\frac{t_{\gamma}^{2}}{4 t}}
\end{aligned}
$$

$C_{\Gamma} \subset \Gamma$ is a complete set of representations in $\Gamma$ of its conjugacy classes, and $C(\gamma)$ is a well defined function on $\Gamma-\{1\}, \rho_{0}=(N-1) / 2$, and $\chi_{\sigma}(m)=\operatorname{trace}(\sigma(m))$ is the character $\sigma$ for $m \in S O(N)$. The trace formula involves the Harish-Chandra-Plancherel measure $\mu_{\sigma_{p}}(r)$ which is given by

$$
\mu_{\sigma_{p}}(r)=\left(\begin{array}{c}
N-1 \\
p
\end{array}\right) \times\left\{\begin{array}{c}
C_{G} \pi P(r) r \tanh (\pi r) \\
\text { for } N=2 n \\
C_{G} \pi P(r), \text { for } N=2 n+1
\end{array}\right.
$$

where $C_{G}=\left(2^{2 N-4} \Gamma(N / 2)^{2}\right)$, and $P(r)$ is a polynomial, which presents the following form

$P(r)=\left\{\begin{array}{l}\prod_{j=0}^{n-2}\left[r^{2}+((2 j+1) / 2)^{2}\right]=\sum_{\ell=0}^{n-1} a_{2 \ell} r^{2 \ell}, \\ \text { for } N=2 n \\ \prod_{j=0}^{n-1}\left[r^{2}+j^{2}\right]=\sum_{\ell=0}^{n} a_{2 \ell} r^{2 \ell}, \text { for } N=2 n+1\end{array}\right.$

$a_{2 \ell}$ are the Miatello coefficients 22, 23]. For $p \geq 1$ there is a measure $\mu_{\sigma}(r)$ corresponding to a general irreducible representation $\sigma$. Let $\sigma_{p}$ be the standard representation of $S O(N-1)$ on $\Lambda^{p} \mathbb{C}^{(N-1)}$. If $N=2 n$ is even then $\sigma_{p}(0 \leq p \leq n-1)$ is always irreducible; if $N=2 n+1$ then every $\sigma_{p}$ is irreducible except for $p=(N-1) / 2=n$, in which case $\sigma_{n}$ is the direct sum of two spin-(1/2) representations $\sigma^{ \pm}: \sigma_{n}=\sigma^{+} \oplus \sigma^{-}$. For $p=n$ the representation $\tau_{n}$ of $K=S O(2 n)$ on $\Lambda^{n} \mathbb{C}^{2 n}$ is not irreducible: $\tau_{n}=\tau_{n}^{+} \oplus \tau_{n}^{-}$is the direct sum of two spin-(1/2) representations.

In the case of the trivial representation ( $p=0$, i.e. for smooth functions or smooth vector bundle sections) the measure $\mu(r) \equiv \mu_{0}(r)$ corresponds to the trivial representation. Therefore, we take $I_{\Gamma}^{(-1)}\left(\mathcal{K}_{t}\right)=H_{\Gamma}^{(-1)}\left(\mathcal{K}_{t}\right)=0$. Since $\sigma_{0}$ is the trivial representation, one has $\chi_{\sigma_{0}}\left(m_{\gamma}\right)=$ 1. In this case, formula (11) reduces exactly to the trace formula for $p=0$ [7, 8, 24, 25].

\section{THE SPECTRAL FUNCTIONS OF EXTERIOR FORMS AND THE VACUUM ENERGY}

If $\mathfrak{L}_{p}$ is a self-adjoint Laplacian on $p$-forms then the following results hold. There exists $\varepsilon, \delta>0$ such that for $0<t<\delta$ the heat kernel expansion for Laplace operators on a compact manifold $X_{\Gamma}$ is given by $\operatorname{Tr}\left(e^{-t \mathfrak{L}_{p}}\right)=$ $\sum_{0 \leq \ell \leq \ell_{0}} a_{\ell}\left(\mathfrak{L}_{p}\right) t^{-\ell}+\mathcal{O}\left(t^{\varepsilon}\right)$. The zeta function of $\mathfrak{L}_{p}$ is the Mellin transform $\zeta\left(s \mid \mathfrak{L}_{p}\right)=(\Gamma(s))^{-1} \int_{\mathbb{R}_{+}} \operatorname{Tr} e^{-t \mathfrak{L}_{p}} t^{s-1} d t$. This function equals $\operatorname{Tr}\left(\mathfrak{L}_{p}^{-s}\right)$ for $s>(1 / 2) \operatorname{dim}(\Gamma \backslash G)$.

The transverse part of the skew-symmetric tensor is represented by the co-exact $p$-form $\omega_{p}^{(C E)}=\delta \omega_{p+1}$, which trivially satisfies $\delta \omega_{p}^{(C E)}=0$, and we denote by $\mathfrak{L}_{p}^{(C E)}$ the restriction of the Laplacian on the co-exact $p$-form. The goal now is to extract the co-exact $p$-form on the manifold which describes the physical degrees of freedom of the system, and presents by alternating sum of forms [26, 27, 28].

\section{A. The identity component of the isometry group}

The zeta function related to the identity integral $I_{\Gamma}^{(p)}\left(\mathcal{K}_{t}\right)$ in (2) has the form

$$
\begin{aligned}
\zeta_{I}\left(s \mid \mathfrak{L}_{p}\right) & =\frac{\chi(1) \operatorname{Vol}(\Gamma \backslash G)}{4 \pi \Gamma(s)} \int_{0}^{\infty} t^{s-1} d t \\
& \times \int_{\mathbb{R}} \mu_{\sigma_{p}}(r) e^{-t y\left(r^{2} ; m_{p}^{2}\right)} d r \\
& =\frac{1}{4} \chi(1) \operatorname{Vol}(\Gamma \backslash G) \\
& \times \int_{\mathbb{R}} \mu_{\sigma_{p}}(r)\left[y\left(r^{2} ; m_{p}^{2}\right)\right]^{-s} d r
\end{aligned}
$$

where $y\left(r^{2} ; m_{p}^{2}\right) \equiv r^{2}+m_{p}^{2}, m_{p}^{2} \equiv b^{(p)}+\left(\rho_{0}-p\right)^{2}$. Because of Eqs. (44) and (5) it is convenient to consider even- and odd-dimensional cases separately.

\section{Even-dimensional manifold}

Using Eqs. (4) and (5) in (6) for $N=2 n$ we get

$$
\begin{aligned}
\zeta_{I}^{(2 n)}\left(s \mid \mathfrak{L}_{p}\right) & =\frac{1}{4} \chi(1) \operatorname{Vol}(\Gamma \backslash G) C_{G} \int_{\mathbb{R}} \frac{P(r) r \tanh (\pi r) d r}{\left[y\left(r^{2} ; m_{p}^{2}\right)\right]^{s}} \\
& =\frac{1}{4} \chi(1) \operatorname{Vol}(\Gamma \backslash G) C_{G} \\
& \times \sum_{j=0}^{n-1} a_{2 j} \int_{\mathbb{R}} \frac{r^{2 j+1} \tanh (\pi r) d r}{\left[y\left(r^{2} ; m_{p}^{2}\right)\right]^{s}} .
\end{aligned}
$$

For $a, \delta>0, z \in \mathbb{C}$, define the entire functions $\mathfrak{K}_{m}(s ; \delta, a) \stackrel{\text { def }}{=} \int_{\mathbb{R}} r^{2 m}\left(\delta+r^{2}\right)^{-s} \operatorname{sech}^{2}(a r) d r$. Then for 
Re $s>j+1, j \geq 0$, one gets [25]

$$
\begin{aligned}
& \int_{\mathbb{R}} \frac{r^{2 j+1} \tanh (a r) d r}{\left(\delta+r^{2}\right)^{s}}= \\
& =\frac{a j !}{2} \sum_{\ell=0}^{j} \frac{\mathfrak{K}_{j-1}(s-\ell-1 ; \delta, a)}{(j-\ell) !(s-1)(s-2) \ldots(s-(\ell+1))}
\end{aligned}
$$

The following result follows:

$$
\begin{gathered}
\zeta_{I}^{(2 n)}\left(s \mid \mathfrak{L}_{p}\right)=\frac{\pi}{8} \chi(1) \operatorname{Vol}(\Gamma \backslash G) C_{\Gamma}\left(\begin{array}{c}
2 n-1 \\
p
\end{array}\right) \sum_{j=0}^{n-1} a_{2 j} j ! \\
\times \sum_{\ell=0}^{j} \frac{\mathfrak{K}_{j-1}\left(s-\ell-1 ; b^{(p)}+\left(\rho_{0}-p\right)^{2}, \pi\right)}{(j-\ell) !(s-1)(s-2) \ldots(s-(\ell+1))} .
\end{gathered}
$$

\section{Odd-dimensional manifold}

In odd-dimensional case, $N=2 n+1$, we get

$$
\begin{aligned}
\zeta_{I}^{(2 n+1)}\left(s \mid \mathfrak{L}_{p}\right) & =\frac{1}{4} \chi(1) \operatorname{Vol}(\Gamma \backslash G) C_{\Gamma}\left(\begin{array}{c}
2 n \\
p
\end{array}\right) \\
& \times \sum_{j=0}^{n} a_{2 j} \int_{\mathbb{R}} r^{2 j}\left[y\left(r^{2} ; m_{p}^{2}\right)\right]^{-s} d r .
\end{aligned}
$$

Using the formula $\int_{\mathbb{R}} r^{2 j+1}\left(\delta^{2}+r^{2}\right)^{-s} d r=$ $\delta^{2 j-2 s} \Gamma(j) \Gamma(s-j) /(\Gamma(s))$, we find:

$$
\begin{gathered}
\zeta_{I}^{(2 n+1)}\left(s \mid \mathfrak{L}_{p}\right)=\frac{\chi(1) \operatorname{Vol}(\Gamma \backslash G)}{4 \Gamma(s)}\left(\begin{array}{c}
2 n \\
p
\end{array}\right) \\
\times \sum_{j=0}^{n} \frac{a_{2 j} \Gamma\left(j+\frac{1}{2}\right) \Gamma\left(s-j-\frac{1}{2}\right)}{m_{p}^{2 s-2 j-1}} .
\end{gathered}
$$

\section{B. The hyperbolic component of the isometry group}

The zeta function associated with hyperbolic orbital integral $H_{\Gamma}^{(p)}\left(\mathcal{K}_{t}\right)$ takes the form

$$
\begin{aligned}
\zeta_{H}\left(s \mid \mathfrak{L}_{p}\right) & =\frac{1}{\sqrt{4 \pi} \Gamma(s)} \sum_{\gamma \in C_{\Gamma}-\{1\}} \chi(\gamma) j^{-1}(\gamma) t_{\gamma} C(\gamma) \\
& \times \int_{0}^{\infty} t^{s-\frac{3}{2}} e^{-t m_{p}^{2}+\frac{t_{\gamma}^{2}}{4 t}} d t
\end{aligned}
$$

Using the $K$-Bessel function $K_{\nu}(s), s \in \mathbb{C}$, defined by $K_{\nu}(2 s) \stackrel{\text { def }}{=}(1 / 2) s^{\nu} \times \int_{0}^{\infty} t^{-\nu-1} \exp \left\{-t-s^{2} / t\right\} d t$, we have

$$
\begin{aligned}
\zeta_{H}\left(s \mid \mathfrak{L}_{p}\right) & =\frac{1}{\sqrt{\pi} \Gamma(s)} \sum_{\gamma \in C_{\Gamma}-\{1\}} \chi(\gamma) j^{-1}(\gamma) C(\gamma) \\
& \times t_{\gamma}^{s+\frac{1}{2}} \frac{K_{-s+\frac{1}{2}}\left(t_{\gamma} m_{p}\right)}{\left[2 m_{p}\right]^{s-\frac{1}{2}}} \\
& =\int_{0}^{\infty} \sum_{\gamma \in C_{\Gamma}-\{1\}} \frac{\chi(\gamma) t_{\gamma} j^{-1}(\gamma) C(\gamma)}{\Gamma(s) \Gamma(1-s)} \\
& \times \frac{e^{-\left(t+m_{p}\right) t_{\gamma}} d t}{\left(2 t m_{p}+t^{2}\right)^{s}}
\end{aligned}
$$

\section{Logarithmic derivative of the Selberg zeta function}

The function $\psi_{\Gamma}(s ; \chi)$ defined in 29

$$
\psi_{\Gamma}(z ; \chi) \stackrel{\text { def }}{=} \sum_{\gamma \in C_{\Gamma}-\{1\}} \chi(\gamma) t_{\gamma} j^{-1}(\gamma) C(\gamma) e^{-\left(z-\rho_{0}\right) t_{\gamma}}
$$

for $\operatorname{Re} s>2 \rho_{0}$, is a holomorphic function in the halfplane $\operatorname{Re} s>2 \rho_{0}$ and admits a meromorphic continuation to the full complex plane. It has been shown that there is a meromorphic function $Z_{\Gamma}(s ; \chi)$ on $\mathbb{C}$ such that $(d / d z) \log Z_{\Gamma}(z ; \chi)=\psi_{\Gamma}(z ; \chi) . Z_{\Gamma}(z ; \chi)$ suitable normalized is the Selberg zeta function attached to $(G, K, \Gamma, \chi)$. Therefore,

$$
\zeta_{H}\left(s \mid \mathfrak{L}_{p}\right)=\frac{1}{\Gamma(s) \Gamma(1-s)} \int_{0}^{\infty} \frac{\psi_{\Gamma}\left(\rho_{0}+t+m_{p} ; \chi\right)}{\left(2 t m_{p}+t^{2}\right)^{s}} d t .
$$

Canonical quantization of Abelian $p$-forms yields a formal expression $(1 / 2) \zeta\left(s=-1 / 2 \mid \mathfrak{L}_{p}\right)=(1 / 2) \sum_{j} \lambda^{1 / 2}$ for the Casimir energy, where $\{\lambda\}_{j \geq 0}^{\infty}$ is the set of eigenvalues (with multiplicity) of the Laplacian $\mathfrak{L}_{p}$ on smooth sections of a vector bundle over $X_{\Gamma}=\Gamma \backslash \mathbb{H}^{N}$ induced by a finite-dimensional unitary representation $\chi$ of $\Gamma$. The regularized Casimir energy related to co-exact forms (the alternating sum of zero- and $p$-form components) on real compact even-dimensional hyperbolic manifolds is given as follows:

$$
\begin{aligned}
E\left(m_{p}\right)_{N=2 n} & =\frac{1}{2} \zeta\left(-1 / 2 \mid \mathfrak{L}_{p}^{(C E)}\right)=\frac{\pi}{16} \chi(1) \operatorname{Vol}(\Gamma \backslash G) C_{G} \\
& \times \sum_{j=0}^{n-1} \sum_{\ell=0}^{j} \frac{a_{2 j} j !}{(j-\ell) ! \prod_{q=0}^{\ell}\left(-\frac{1}{2}-(q+1)\right)} \\
& \times\left[\left(\begin{array}{c}
2 n-1 \\
p
\end{array}\right) \mathfrak{K}_{j-1}\left(-\ell-\frac{3}{2} ; m_{p}^{2}, \pi\right)\right. \\
& \left.+\mathfrak{K}_{j-1}\left(-\ell-\frac{3}{2} ; m_{0}^{2}, \pi\right)\right] \\
& -\frac{1}{2 \pi} \int_{0}^{\infty}\left\{\psi_{\Gamma}\left(\rho_{0}+t+m_{p} ; \chi\right)\left[2 t m_{p}+t^{2}\right]^{\frac{1}{2}}\right. \\
& \left.+\psi_{\Gamma}\left(\rho_{0}+t+m_{0} ; \chi\right)\left[2 t m_{0}+t^{2}\right]^{\frac{1}{2}}\right\} d t . \quad(16)
\end{aligned}
$$


In the case of scalar field ( $p=0$ for a trivial representation) the Casimir energy becomes

$$
\begin{aligned}
& E\left(m_{0}\right)_{N=2 n}=\frac{\pi}{16} \chi(1) \operatorname{Vol}(\Gamma \backslash G) C_{G} \\
& \times \sum_{j=0}^{n-1} \sum_{\ell=0}^{j} \frac{a_{2 j} j ! \mathfrak{K}_{j-1}\left(-\ell-\frac{3}{2} ; m_{0}^{2}, \pi\right)}{(j-\ell) ! \prod_{q=0}^{\ell}\left(-\frac{1}{2}-(q+1)\right)} \\
& -\frac{1}{2 \pi} \int_{0}^{\infty} \psi_{\Gamma}\left(\rho_{0}+t+m_{0} ; \chi\right)\left[2 t m_{0}+t^{2}\right]^{\frac{1}{2}} d t
\end{aligned}
$$

Formula (16) with positive parameter $m_{p}$ gives the regularized vacuum energy $E\left(m_{p}\right)$ which is finite. From (11) it follows that in the case of odd $N$ the identity component of $E\left(m_{p}\right)$ has poles at $s=-1 / 2$ and therefore $E\left(m_{p}\right)$ cannot be obtained by the method available for even-dimensional manifolds, which agrees with result obtained in [13]. For the trivial representation $\chi=1$ of $\Gamma$, the topological component of the Casimir energy $E\left(m_{p}\right)$ (the last term in (16)) is always negative, in agreement with results previously obtained in 14]. In the case of scalar fields (zero-forms) our result agrees with one founded in 13 .

\section{CONCLUDING REMARKS}

Cosmological predictions, such as the microwave background anisotropies (CMB) and the current acceleration expansion of the universe [30], depend pretty much on the details of the theoretical model under consideration.
In particular, the recent data obtained by the Wilkinson Microwave Anisotropy Probe (WMAP) 31] satellite confirmed, and set new standards of accuracy, to the previous COBE's measurement of a low quadrupole moment in the angular power spectrum of the CMB, which is in accordance with the assumption that the topology of the universe might be non-trivial, with particular emphasis on the case of a compact hyperbolic universe. Combined with this observation, the WMAP satellite also indicates that $\sim 60 \%$ of the critical energy density of the universe is contributed by a smoothly distributed vacuum energy (Casimir energy) or dark energy, whose net effect is repulsive (leading, thus, to an accelerated expansion of the universe).

In this paper, we have shown that the topological component of the Casimir energy for co-exact forms on evendimensional manifolds, associated with the trivial character, is always negative. This result confirms the above mentioned measurements and we can infer on the cosmological consequences of it. We plan to address this question in details in a forthcoming paper [32].

\section{Acknowledgements}

A. A. Bytsenko and M. E. X. Guimarães would like to thank the Conselho Nacional de Desenvolvimento Científico e Tecnológico (CNPq/Brazil) for partial support. V. S. Mendes would like to thank CAPES for a PhD grant.
[1] B. De Witt, Phys. Rep.19, 295 (1975).

[2] N. Birrell and P. W. Davis, Quantum Fields on Curved Soaces (Cambridge University Press, Cambridge, 1982).

[3] J. Ambjorn and S. Wolfram, Ann. Phys. (N.Y.)147, 1 (1983).

[4] W. Greiner, B. Muller and G. Plunien, Phys. Rep. 134, 87 (1986).

[5] A. Jackson and L. Vepstas, Phys. Rep. 187, 109 (1990).

[6] V. Mostepanenko and N. Trunov, Casimir Effects and Its Applications (Energoatomizdat, Moscow, 1990).

[7] E. Elizalde, S. D. Odintsov, A. Romeo, A. A. Bytsenko and S. Zerbini, Zeta Regularization Techniques with Applications (World Scientific, Singapore, 1994).

[8] A. A. Bytsenko, G. Cognola, L. Vanzo and S. Zerbini, Phys. Rep. 266, 1 (1996).

[9] A. A. Bytsenko, G. Cognola, E. Elizalde, V. Moretti and S. Zerbini, Analytic Aspects of Quantum Fields (World Scientific, Singapore, 2003).

[10] A. A. Bytsenko and Yu. P. Goncharov, Class. Quantum Grav. 8, 2269 (1991).

[11] A. A. Bytsenko and Yu. P. Goncharov, Class. Quantum Grav. 8, L211 (1991).

[12] A. A. Bytsenko and Yu. P. Goncharov, Mod. Phys. Lett. A 6, 669 (1991).

[13] A. A. Bytsenko and S. Zerbini, Class. Quantum Grav. 9, 1365 (1992).
[14] G. Cognola, L. Vanzo and S. Zerbini, J. Math. Phys. 32, 222 (1992).

[15] G. Cognola, K. Kirsten and S. Zerbini, Phys. Rev. D 48, 790 (1993).

[16] M. J. Duff, B. E. Nilsson and C. N. Pope, Phys. Rep. 130, 1 (1986).

[17] J. Maldacena, Adv. Theor. Math. Phys. 2, 231 (1998).

[18] E. Elizalde, e-Print arXiv: hep-th/0311195

[19] R. Aurich, S. Lustig, F. Steiner and H. Then, e-Print arXiv: astro-ph/0403597

[20] N. Kaloper, J. March-Russell, G. D. Starkman and M. Trodden, Phys. Rev. Lett. 85, 928 (2000).

[21] D. Fried, Invent. Math. 84, 523 (1986).

[22] R. Miatello, Trans. Am. Math. Soc. 260, 1 (1980).

[23] A. A. Bytsenko, E. Elizalde and M. E. X. Guimarães, Int. J. Mod. Phys. A 18, 2179 (2003).

[24] N. Wallach, J. Diff. Geom. 11, 91 (1976).

[25] A. A. Bytsenko and F. L. Williams, J. Math. Phys. 266, 1075 (1998).

[26] A. A. Bytsenko, L. Vanzo and S. Zerbini, Nucl. Phys. B505, 641 (1997).

[27] A. A. Bytsenko, Nucl. Phys. (Proc. Suppl.) B104, 127 (2002).

[28] A. A. Bytsenko, A. E. Gonçalves and F. L. Williams, Int. J. Mod. Phys. A 18, 2041 (2003).

[29] R. Gangolli, Ill. J. Math. 21, 1 (1977). 
[30] S. Perlmutter et al., Ap. J. 517, 565 (1999).

in preparation.

[31] H. V. Peiris et al., Ap. JS 148, 213 (2003).

[32] A. A. Bytsenko, M. E. X. Guimarães and V. S. Mendes, 